\title{
Messengers of the Universe
}

\author{
F. Donato ${ }^{\mathrm{a}}$ J. Knapp ${ }^{\mathrm{b}}$ \\ ${ }^{a}$ Physics Department, Torino University and INFN, via Giuria 1, 10125 Torino, Italy \\ ${ }^{b}$ Deutsches Elektronen-Synchrotron, Platanenallee 6, 15712 Zeuthen, Germany
}

\begin{abstract}
The past two years have seen momentous changes and new results in Astroparticle Physics: AMS and Pamela have provided measurements of unprecedented statistical precision of the spectra of various types of cosmic rays with unprecedented statistics and precision. Some of theses spectra show rather intriguing features which are topics of ongoing debate. For the first time, neutrinos with energies up to $10^{15} \mathrm{eV}$ have been detected, which must be of extra-terrestrial origin. The number of $\gamma$-ray sources has surpassed 150, with $\mathrm{E}_{\gamma}>100 \mathrm{GeV}$, and 3000, with $\mathrm{E}_{\gamma}>100$ $\mathrm{MeV}$. They belong to a variety of source classes, with many of the sources still being unidentified. With such numbers population studies become feasible, and the the understanding of acceleration mechanisms can be steadily increased. In interactions of relativistic, charged particles $\gamma$-rays and neutrinos are produced as secondaries, with somewhat lower energies. Therefore, high-energy cosmic rays, photons and neutrinos, originate likely from the same sources. Thus, the era of multi-messenger observations and analyses has truly arrived. New results pose constraints to a number of models and stimulate the whole field. This is a summary of the parallel presentations from Session IV: "Messengers of the Universe" of the Neutrino Oscillation Workshop 2014.
\end{abstract}

Keywords: Cosmic Rays, High-Energy Gamma Ray Astronomy, Neutrino Astronomy, Cosmic Accelerators

\section{Introduction.}

In Session IV: "Messengers from the Universe" of the NOW 2014, the scientific breadth of astroparticle physics has been well demonstrated with excellent reviews in the respective plenary sessions by Koskinen $^{1}$ (IceCube \& PINGU), Sapienza (KM3NeT),

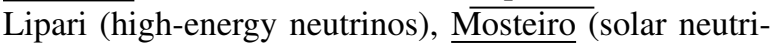

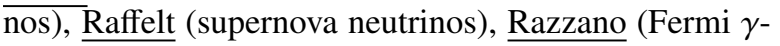
ray results) and Waxman (multi-messenger astronomy). In many respects, the radiation reaching Earth in the energy range of tens of $\mathrm{MeV}$ up to $10^{20} \mathrm{eV}$ is still a mystery. Over the past decade, this has triggered a wealth of activity in the field with many results adding up to a good and steady progress. Often, the relation and interplay between cosmic rays (CR), photons of various

\footnotetext{
${ }^{1}$ Underlined names refer to other papers in these proceedings and to the corresponding presentations on the conference webpage http://www.ba.infn.it/ now/now2014
}

energies and neutrinos is at the core of investigations, combining observations from various particle types and different wavelengths. This so-called multi-messenger approach becomes increasingly important and fruitful, allowing more encompassing studies of the properties of cosmic sources of high-energy particles. The violent environments in which the high-energy particles are produced are exposing matter and interactions at extreme conditions. Merging and accreting compact objects, such as black holes and neutron stars, relativistic jets and particle winds, highly variable active galactic nuclei, supernova explosions and $\gamma$-ray bursts, rapidly spinning pulsars with enormous magnetic fields, are all seen to produce high-energy emission. Therefore, highenergy particles are well suited to study these objects and to probe our knowledge of the physics in extreme conditions. The analyses and results presented in the parallel sessions on "Messengers from the Universe" are summarised in the following chapters. 

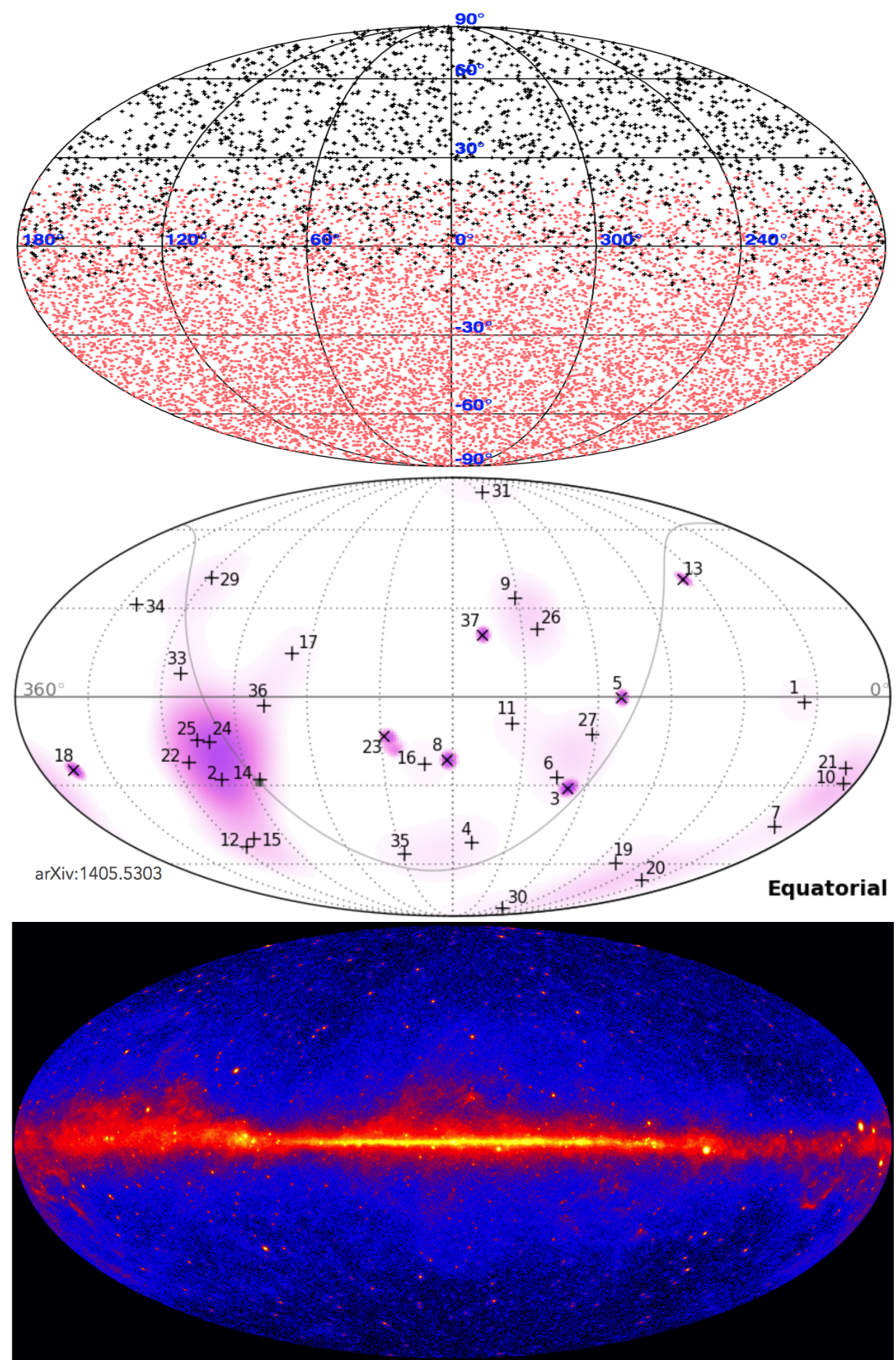

Figure 1: The sky in high-energy particles. Top: CRs with $\mathrm{E}>10 \mathrm{EeV}$ from the Pierre Auger Observatory and the Telescope Array. Middle: neutrinos with $\mathrm{E}>20 \mathrm{TeV}$ from IceCube. Bottom: $\gamma$-rays with $\mathrm{E}>100 \mathrm{MeV}$ from the Fermi-LAT instrument. CRs and neutrinos are shown in equatorial, $\gamma$-rays in galactic coordinates. CRs suffer deflection in cosmic magnetic fields, which may be large enough to obscure arrival directions, even for $\mathrm{E}>10 \mathrm{EeV}$. 


\section{Cosmic Rays}

The measurement of the CR energy spectrum shows a power-law, with slightly varying spectral index through the whole energy range. These features occur at about $3 \times 10^{15} \mathrm{eV}$ (the so-called knee) and around $3 \times 10^{18}$ $\mathrm{eV}$ (the ankle). The particles detected below the knee are supposed to have been accelerated by galactic supernova remnants (SNRs), while the highest energy spectrum is of less clear origin. Nevertheless, it is generally accepted that the accelerators of particles with $\mathrm{E}>10^{18}$ $\mathrm{eV}$ must be of extragalactic origin. CRs being charged suffer from deflection,

Aloisio has reviewed models for the production and propagation of the ultra-high energy (UHE) particles, and their comparison with HiRes, Telescope Array (TA) and Auger data. The data from HiRes and TA are compatible with an UHE universe dominated by protons, with a transition galactic/extragalactic energy located around $10^{18} \mathrm{eV}$ and a possible maximal energy exceeding $10^{20} \mathrm{eV}$. The Auger data, with much larger statistics and rather well understood systematics, indicate that the highest-energy $\left(>4 \times 10^{18} \mathrm{eV}\right)$ CRs contain many heavy nuclei, accelerated according to a quite flat injection spectrum. For the diffuse spectra, the HiRes/TA scenario would predict fluxes of $\gamma$-rays and neutrinos just within the reach of Fermi-LAT, IceCube, and Auger detectors (see also Bleve). In the Auger scenario, however, very low, almost undetectable fluxes are expected. PeV neutrinos are predicted from the interaction of the small UHE proton tail with the extragalactic background light. As for source spectra, the observed $\gamma$-emission from active galactic nuclei (AGN) can be interpreted in terms of the secondary $\gamma$-rays produced by UHECRs interacting with the CMB.

As for CRs of galactic origin, detected at much lower energies, we have had talks about the two most recent space-based experiments. Mori has presented the CR observations by the Pamela experiment, which has been taking data for 8 years and until the end of 2015. The main scientific results are the rise in the positron fraction, indicating a primary component, spectral features in the $\mathrm{H}$ and $\mathrm{He}$ spectra, the discovery of geomagnetically trapped antiprotons, and the absence of peculiar spectral features in the cosmic antiproton flux, indicating the absence of a primary component. Forthcoming results from Pamela include the measurement of the fluxes of nuclei up to oxygen, anisotropy studies, time dependent data for $e^{ \pm}$and $p$ fluxes, and the detection of solar events. In the talk by Tomassetti, the major physics results and challenges foreseen for the AMS-02 experiment have been outlined. AMS-02 is a the major space- borne CR detector, installed on the International Space Station and conceived for high-precision studies of CRs up to $\mathrm{TeV}$ energies. It is shown in particular that the measured positron fraction increases steadily from 10 to $250 \mathrm{GeV}$, confirming the Pamela data with higher precision. Data on absolute leptons, proton, helium and the boron-to-carbon ratio have also been presented. A theoretical interpretation of the preliminary AMS-02 data on the lepton fluxes has been discussed by Fornengo. The data are interpreted in terms of different known astrophysical sources, such as supernova remnants, pulsars and econdary particles produced by the interactions of hadronic CRs in the Galaxy. A remarkable agreement with all the data is found, both for an average pulsar population and for a single or very few powerful pulsars nearby.

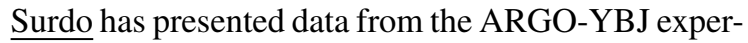
iment, which measured the CR spectrum in the $\mathrm{TeV}$ to 5 $\mathrm{PeV}$ energy range. The all-particle spectrum is consistent with previous measurements. Preliminary results, obtained from three different analyses, for the $\mathrm{p}+\mathrm{He}$ spectrum show a bending below $1 \mathrm{PeV}$. This would be an important input for an improved estimation of the atmospheric $v$ flux.

\section{Gamma Rays}

In the past few years, $\gamma$-ray astronomy has been boosted through many results from the three groundbased $\gamma$-ray observatories H.E.S.S., MAGIC and VERITAS, and the simultaneous operation of $\gamma$-ray detectors aboard satellites (Fermi, AGILE and SWIFT). The combination of their data with data of radio, and X-ray instruments yielded a wealth of new results on numerous sources of non-thermal radiation in our Galaxy and beyond. $\gamma$-rays are ubiquitous messengers from electromagnetic processes, travel in straight lines, and are (relatively) easy to detect. More than 150 well established sources in the $\mathrm{TeV}$ range and more than 3000 in the $\mathrm{GeV}$ range are testament to the great progress during get the past decade. Therefore, $\gamma$-rays are the prime messengers to study cosmic particle accelerators in detail and to learn about the acceleration mechanisms at work.

Razzano summarised the impressive harvest of the Fermi satellite from its six years of operation in the plenary session. Most notably, the $\gamma$-ray sky turned out to be rich and dynamic, with 23000 sources ranging from terrestrial $\gamma$-ray flashes to AGN and GRBs at cosmological distances, and a diffuse background from weak sources and the interactions of CRs with ambient matter. The most abundant source classes are AGN, SNR, 
pulsars and pulsar wind nebulae (PWN), and unidentified sources. Totally unexpected phenomena have been found, like the still poorly understood Fermi bubbles and the terrestrial $\gamma$-ray flashes. Also, using the Earth magnetic field, the electron and positron spectra could be measured, complementing dark matter searches with photons.

Bleve reviewed the searches for $\gamma$-rays (and neutrinos) among the UHE CRs recorded with the Pierre Auger Observatory. The much more abundant events from the surface array are now used for $\gamma$-ray searches and the analysis has been much refined. The result are the most stringent limits for UHE photons. Between $1-4 \times 10^{19} \mathrm{eV}$ are closing in on region where photons from the GZK reaction can be expected for $\mathrm{p}$-dominated scenarios. The same is true for hybrid (surface array and fluorescence telescopes) analyses. Especially near $2 \times 10^{18} \mathrm{eV}$ the limits are closest to predictions from the GZK reactions. Z-bursts as a source of UHE $\gamma$-rays are firmly excluded. In (blind) directional searches for point sources of UHE $\gamma$-rays no significant excess could be found. The average upper limit (95\% CL) for steady sources is 0.035 photons $/\left(\mathrm{km}^{2} \mathrm{yr}\right)$ with a maximum of 0.14 photons $/\left(\mathrm{km}^{2} \mathrm{yr}\right)$.

Valerius presented an overview of SNRs and pulsar wind nebulae (PWN), extensively studied with current $\gamma$-ray observatories. They are prime examples of cosmic particle accelerators. Their power comes from the explosion and from rapid pulsar rotation and acceleration can occur at shock fronts as well as in the pulsar magnetosphere, for electrons and hadrons. At TeV energies they are, luckily, one of the largest classes of sources and objects are seen in various stages of their lives. Correlation with pulsar data of all wavelengths and population studies help greatly to classify, model and understand these sources. The evidence strengthens that young SNRs ( $<$ few 100s of years) can produce, in their shells, the PeV particles in our galaxies. For PWN, a correlation of their detection in $\gamma$-rays with their spindown rate has been observed pointing to rotating magnetic fields as accelerators. Multi wavelength studies allow tentatively to separate the leptonic and hadronic origin of $\gamma$-rays. With the planned Cherenkov Telescope Array (CTA), which will provide ten times more sources, these investigations will reach new levels of understanding.

The presentation of Calore contributed to this topic by studying milli second pulsars (MSPs) from the radio (ATNF catalog) to $\mathrm{GeV}$ (Fermi-LAT) range, The aim was to describe part of the diffuse $\gamma$-ray emission (e.g. the Fermi GeV excess) as coming from the MSP population. So far, young pulsars and MSPs can only account for about $10 \%$ of the $\mathrm{GeV}$ excess for small galactic latitudes. Their contribution to the isotropic $\gamma$-ray background and the $\gamma$-ray anisotropy seen by Fermi-LAT is marginal.

Star forming galaxies throughout the universe can contribute to the diffuse $\gamma$-ray and neutrino background. Tamborra presented an analysis where emission form star forming galaxies, with a suitable spectral slope, match simultaneously the Fermi $\gamma$-ray spectrum in the $100 \mathrm{MeV}$ to $100 \mathrm{GeV}$ range and the IceCube neutrino spectrum in the $6 \times 10^{12}$ to $10^{15} \mathrm{eV}$.

$\gamma$-rays provide not only precious information on particle acceleration and high-energy astrophysics of numerous cosmic objects, they allow also tests of key principles of modern physics, such as the test of Lorentz invariance. Daniel illustrated how current and especially future $\gamma$-ray observatories, such as CTA, can improve these tests. Many models of Quantum Gravity suggest an energy dependent speed of light, which would violate Lorentz invariance. Rapidly variable emission over a wide energy range from distant sources is required for these tests and the best current limits come from GRB observations with Fermi. Also flaring AGN have led to stringent limits. CTA will have a far better sensitivity for variability at energies $>10 \mathrm{GeV}$ and will observe many variable sources, so that the violation of Lorentzinvariance may be detected convincingly.

\section{Neutrinos}

The Sun is one of the only two well-established astrophysical neutrino sources. Just recently Borexino has published an impressive detailed measurement of the primary $p$-p fusion process in the Sun and Mosteiro presented this result in the plenary session. The result is foremost the measurement of the $p$-p neutrino flux and the oscillation behaviour, but also a stringent test of solar variability (on the $10^{5} \mathrm{yr}$ level) and the Standard Solar Model. About 30 astrophysical (MeV) neutrinos have been detected from supernovae 1987a in the Large Magellanic Cloud, but since then the neutrino community is waiting eagerly with largely improved detectors for neutrino signals from other supernova explosions. Is is only a matter of time until they are rewarded. Just recently, IceCube has published the first solid evidence for high-energy astrophysical neutrinos. At energies of $60 \mathrm{TeV}$ to $2 \mathrm{PeV}$ they found 35 events, which cannot be explained by atmospheric background. Thus, for the first time astrophysical neutrinos have been seen with energies above a few $\mathrm{MeV}$, and in a range of highest importance for high-energy and non-thermal astrophysics. Unfortunately, so far the neutrinos cannot be associated 
to sources. They are sufficiently scattered over the sky, not to exclude an isotropic distribution, and no significant clustering is seen so far. With more data the source search will improve, but as IceCube used already the date from 3 years, statistical improvement with the same detector will be slow. Many attempts are undertaken to correlate the neutrino directions with potential source populations, so far without convincing results. A quite disappointing possibility could be that the fluxes from individual neutrino sources are so small that no more than one neutrino is observed from any one source. In this case the association with a source population would become very difficult and the neutrinos would remain a truly diffuse background, rather similar to the situation faced currently by UHECR experiments at E $>10^{19} \mathrm{eV}$.

Searches for UHE neutrinos with the Pierre Auger Observatory (Bleve) use now data from 2004-2012. So far no neutrino candidates have been found, neither in the Earth skimming, not in the downward going sample of events. Limits in the energy range $10^{17}$ to $2 \times 10^{19}$ $\mathrm{eV}$ are for the first time lower than the Waxman-Bahcall limits and compare well with limits from IceCube for $<4 \times 10^{18} \mathrm{eV}$. The limits start to constrain the area where neutrinos are expected for p-dominated scenarios, but are still more than an order of magnitude above what Fe-dominated scenarios suggest. Further improvements in the limits may come from advances in the data analysis and the extensions of the Auger surface detectors for improved muon identification. A factor 2-3 may still be possible in the next decade.

Margiotta presented a status report of ANTARES, the first undersea neutrino telescope and he largest in the Northern hemisphere. With its favourable view on the galactic centre, and excellent angular resolution and the long exposure of $\sim 1400$ days, and despite its small size, it provides currently the best limits for Galactic sources. Searches for source have not produced any significant results, so far. ANTARES runs an extensive multimessenger program with experiments on $\mathrm{GeV}$ and $\mathrm{TeV}$ $\gamma$-rays, UHECRs, optical and X-ray photons and gravitational waves to enhance chances for a significant detection while waiting for the advent of KM3NeT in the Mediterranean sea.

Resconi reported on a study of $\gamma$-ray counterparts to the 35 IceCube astrophysical neutrino events with energies $60 \mathrm{TeV}-2 \mathrm{PeV}$. They must originate from primary CRs of about $1-40 \mathrm{PeV}$. This is the region of the "knee" in the CR energy spectrum, which is generally attributed to the end of the galactic CR population, and an energy where many extragalactic accelerators operate. It is inconceivable that sources producing so energetic CRs (PeVatrons) are not also producing many $\gamma$-rays in the range up to $100 \mathrm{TeV}$, thus, the search for $\gamma$-rays counterparts, even though $\gamma$-rays from distant sources might suffer considerable absorption. With a large number of plausibel counterparts (galactic and extragalactic) close to the level of the detected neutrino flux, an identification of sources seems immanent. IceCube is just at the sensitivity threshold of a new cosmic landscape.

Zuber presented first results from the HALO detector, a setup made of lead target and ${ }^{3} \mathrm{He}$ neutron counters, located underground at SNOLab. $v_{e}$ and $v_{x}$ would react with the lead via charge current and neutral current reactions, respectively, leading to 1-n and 2-n emission in subsequent decays. The neutrons are captured in the ${ }^{3} \mathrm{He}$ detectors. Neutron bursts (e.g. from supernova explosions) over the steady neutron background are searched for since summer 2013 (so far without success). HALO is integrated into the Supernova early warning system (SNEWS). For the future, an improved and enlarged $1 \mathrm{kt}$ lead detector (HALO-2) is discussed.

The hierarchy of the masses of the three known neutrinos is still not established, given that oscillation experiments give usually only information on the squared mass differences. Kappes has shown very didactically how matter effects in oscillations of few-GeV atmospheric neutrinos together with a $30 \%$ asymmetry of neutrinos and anti-neutrinos, allow the determination of the ordering of the neutrino mass eigenstates. He also discussed how the possible theoretical systematics can be reduced, using forthcoming data from ORCA and PINGU. It is shown that the experimental sensitivity depends notably on the value of $\theta_{23}$, and is maximal for this angle lying in the second octant $\left(>45^{\circ}\right)$.

The talk by Stanev dealt with the computation of the high-energy atmospheric neutrino flux. In particular, he discussed the performance of the new (not yet released) version of the hadronic interaction model SIBYLL 2.XX that also generates hadrons with charm, which decay and generate very high-energy atmospheric neutrinos. The interest in the charm channel for neutrino production is motivated by the detection by IceCube of a number of neutrinos of energy above $30 \mathrm{TeV}$, a fraction of which must be of astrophysical origin. The contribution of charm to the atmospheric neutrino flux at higher energies leads to a firmer prediction for the flux of astrophysical neutrinos. Predictions are tested against laboratory data from various experiments at fixed targets and at the LHC. The release of the new SIBYLL code, containing prompt neutrinos from charmed particles, should be ready soon.

A discussion on the possible origin of the diffuse neutrino flux detected by Icecube as been performed by Taylor. The IceCube high energy astrophysical 
neutrinos were analysed in a Galactic origin context. Emission scenarios from both, the Fermi bubble and a broader halo region, have been considered. Taylor showed that the Fermi bubbles may be an indicator of cosmic ray outflow, following activity in the Galactic center. Also, the expected Galactic plane emission can be outshone by the Galactic halo, provided the halo is sufficiently big and contains enough target material. The current appearance of the bubbles suggests a strong accelerating source (a PeVatron,)in the Galactic Centre, the detection of which in $\gamma$-rays would be crucial for helping to understand the IceCube neutrino signal which seems to have some enhancement not far from the Galactic Centre.

\section{Outlook}

The future of astroparticle physics is bright. For cosmic rays, $\gamma$-rays and neutrinos new experiments are planned to largely extend their performance. The Pierre Auger Observatory is enhanced for better muon identification which will help mass composition studies in the energy range $>10^{17} \mathrm{eV}$. The Cherenkov Telescope Array (CTA), with two observatories, one on each hemisphere, will significantly increase the accessible energy range for $\gamma$-rays $(20 \mathrm{GeV}$ to $300 \mathrm{TeV})$ and improve the sensitivity, angular and energy resolution by about an order of magnitude compared to existing observatories. Especially, for short-timescale phenomena its sensitivity will be several orders of magnitude better than that of the Fermi satellite. Of the order of 1000 new $\gamma$ ray sources should be detectable with this instrument, populations studies of sources will become feasible and many galactic sources will be resolved. HAWC, an allsky survey instrument with a somewhat higher energy threshold than CTA has started operation in Mexico, and will produce interesting science in due time. While, IceCube and Antares are taking data, both teams plan for considerable extensions. The IceCube Collaboration will extend its detector from $1 \mathrm{~km}^{3}$ to about $10 \mathrm{~km}^{3}$, in order to collect more statistics of the high-energy, astrophysical neutrinos, in the hope to identify their sources, either by population studies or by seeing clusters in the sky. ANTARES aims to build KM3NeT in the Mediterranean sea. This would be an instrument of similar capabilities as IceCube, yet with the prime view on our Galactic Centre. Both Collaborations, on the other hand, plan to deploy very densely packed photosensors in the centre of their neutrino detectors to lower the neutrino detection thresholds to the level of few GeV. This would allow oscillations studies of atmospheric neutrinos from which the yet enigmatic mass hierarchy of neutrinos could be determined, at a fraction of the cost of dedicated beam or reactor experiments, and possibly at a much shorter timescale.

Not only the experiments become more sensitive and sophisticated, also the connections between them grow rapidly stronger. No analysis is nowadays complete without the study of counterparts in other particle types and wavelengths. For now, CRs, neutrinos and photons (from radio to $\gamma$ rays) are the main messengers considered, but soon also gravitational waves may become part of the signals which can be used to do highenergy astrophysics. Especially for transients, the simultaneous observation of a source in different and independent channels is greatly improving the sensitivity of an observation. One initiative in this direction is the Astrophysical Multi-messenger Observatory Network (AMON; http://amon.gravity.psu.edu). It is a program currently under development in collaboration with a growing list of observatories. AMON aims at realtime correlation analyses of high-energy signals across all known astrophysical messengers. It is to enable mutual alerting and rapid follow-up analyses of putative astrophysical signals. Thus, the "Messengers of the Universe" and their connections offer exciting prospects for the decade ahead and will certainly feature prominently on future conferences of the NOW series.

\section{Acknowledgments}

We would like to thank G. Fogli and E. Lisi for their invitation and the pleasant and stimulating atmosphere of the NOW2014 workshop. 